\title{
INFLUENZA VACCINATION RATE AMONG HIGH RISK GROUP PATIENTS IN PRIMARY HEALTH CARE IN GREECE
}

\author{
Anastasia Papaioannou ${ }^{1,2}$, Apostolia-Evangelia Konstantinidi ${ }^{2}$, Ekaterini Primikiri², Foteini Asimakopoulou ${ }^{2}$, \\ Dimitrios Aravantinos ${ }^{2}$, Zacharoula Mavromichali² \\ ${ }^{1}$ Health Centre of Nea Makri, Athens, Greece \\ ${ }^{2}$ Health Centre of Kapandriti, Athens, Greece
}

\section{SUMMARY}

Objective: Seasonal influenza vaccination is the main method for influenza prevention. The main objective of this study is to estimate the frequency of vaccinations in patients with chronic illnesses presented to a primary health care (PHC) centre.

Methods: This cross-sectional study was performed in patients admitted to the Kapandriti Health Centre. Their vaccination status with an influenza vaccine and their underlying diseases were recorded.

Results: $34.8 \%$ of the subjects had been vaccinated against seasonal influenza. Vaccination coverage was found to be $53.9 \%$ in pulmonary, $55.6 \%$ in chronic kidney disease, $43.7 \%$ in cardiovascular disorders, $40.6 \%$ in diabetes, $40.6 \%$ in any kind of malignancy, and finally $33.3 \%$ in neurological patients. The most significant predictors for vaccination were the age group of 60 to 79 years (OR=3.08, 95\% $\mathrm{Cl}: 1.79-5.29)$, age over 80 years $(\mathrm{OR}=2.91,95 \% \mathrm{Cl}: 1.58-5.36)$, respiratory disease ( $\mathrm{OR}=2.25,95 \% \mathrm{Cl}: 1.33-3.76)$, cardiovascular disorder $(\mathrm{OR}=1.46,95 \% \mathrm{Cl}$ : $1.02-2.10)$, and 3 to 5 visits to the unit annually $(\mathrm{OR}=1.57,95 \% \mathrm{Cl}: 1.12-2.24)$. Finally, it was discovered that coexistence of one to three diseases reduced the likelihood ratio for vaccine uptake $(\mathrm{OR}=0.15,95 \% \mathrm{Cl}: 0.03-0.79, \mathrm{p}<0.05)$.

Conclusions: The influenza vaccination rate for the population of the present study has been found higher than that reported previously in literature. We believe that there is a need to implement new and more effective strategies such as educating vulnerable groups on the benefits of vaccination and so reducing the incidence of influenza and its complications especially in vulnerable groups.

Key words: influenza, vaccination, high risk group, primary healthcare, Greece

Address for correspondence: A. Papaioannou, Health Centre of Nea Makri, Marathonos Av. and Artemidos, Nea Makri, 19005, Athens, Attica, Greece. E-mail: anpapai@yahoo.com

https://doi.org/10.21101/cejph.a5237

\section{INTRODUCTION}

Influenza is a major public health problem that greatly affects the mortality and morbidity of the population and it is a significant stretch factor for global healthcare services. The flu virus is responsible for approximately 40,000 deaths in Europe annually, with the majority of victims being the elderly with underlying chronic diseases (1). Adults older than 65 years, individuals with underlying medical conditions and pregnant women are considered at high risk of severe disease or complications (2). Vaccination for seasonal influenza is the most effective preventive measure and is recommended annually to vulnerable population groups. Previous estimations showed that more than 120 million adults in the European Union (EU) belong to one or more high risk groups that could potentially benefit from vaccination (3). Vaccination efficacy is proportional to the antigenic matching between the vaccine and the circulating strain, as well as other factors such as the time of vaccination and the presence of other respiratory tract viruses (4). In 2003, the World Health Assembly adopted Resolution WHA56.19 urging Member States to increase influenza vaccination coverage of all people at high risk and to attain coverage of $75 \%$ among the elderly by 2010 (5).
Several countries routinely conduct a survey to estimate the effectiveness of annual vaccination rate $(6,7)$. However, such studies fail to clearly indicate the percentage of vaccination coverage in high risk groups. In addition, data publications at primary health care level are even fewer.

The National Hellenic Immunization Programme (NIP) is based on the recommendations of the National Vaccine Commission in agreement with the Greek Ministry of Health. Vaccines are prescribed to Greek citizens, including immigrants/refugees, mainly in primary health care, free of charge. Unfortunately, there is no national registration log, nor a formal reminder system (8).

Pharmacists in Greece can in several cases effectively administer influenza vaccinations. This strategy is also promoted in the United Kingdom, Canada and the United States, and has been found to increase immunization rates and meet service user needs (9-11). Thus, the coverage rates are unknown and the only way to obtain such information is through the medical provider requesting such details from the individual with a low possibility to accrue national level data.

Regarding Primary Health Care (PHC) in Greece, it appears that there is no official data on vaccination rate of patients in high risk groups with severe complications. Hence, the aim of the 
present study was to determine the influenza vaccination status in high risk adults, within PHC in Greece. The primary outcome was influenza vaccination during the past 12 months.

\section{MATERIALS AND METHODS}

A cross-sectional study was performed to determine the influenza vaccination rate of patients over 60 years, presented for periodic health evaluation and medical care to the Health Centre of Kapandriti, Athens, from May 2016 to May 2017.

The Health Centre of Kapandriti is located $30 \mathrm{~km}$ from the Athens city centre, in a suburban region of Attica. Medical services are available on a 24-hour basis and include emergency medical care, medical counselling, evaluation and prevention for adults and children, as a primary health care unit. The unit provides medical and follow-up care for chronic diseases. These services are mostly provided by general/family doctors, paediatricians, nurses and social workers, while a number of specialized practitioners are also included in the units' workforce.

The studied population included individuals older than 60 years and/or adults with a high risk for post-influenza complications. Greek immunization protocol recommends to individuals over 60 years vaccination for influenza every year. Data was collected from the medical history of patients that presented in the PHC unit on regular days. In addition, patients were asked if they had been vaccinated for influenza, either in the previous or current season, as well as if they were smokers. People with diagnosed diabetes mellitus, cardiovascular disease, respiratory disease, neurological conditions, and people with any kind of malignancy, while on medication, were considered as high risk patients. Pregnant women were excluded. Other exclusion criteria were language barriers with immigrants or mental disability.

The following background characteristics were exported: age, gender, chronic medical condition, multimorbidity, health insurance, place of habitation, annual number of visits to the unit and smoking status. The main variable was vaccination rate in adulthood. Vaccination rate in adulthood was defined as the percent of patients in high risk group who were vaccinated against influenza to the overall number of presenting patients.

\section{Sample Size}

Sample size was calculated using the sample size formula for estimating minimum sample size in a cross-sectional study as described previously (12). For this calculation a type I error probability associated with the test of the null hypothesis of 0.05 and a power of $80 \%$ was applied. Using a vaccination rate of $14.8 \%$ $(0.148)$ as mean percentage of vaccination from previous studies, the minimum sample size required for this study was estimated to be 194 . Nevertheless, data from all 1,168 patients were eventually included in the study in order to increase the sampling precision.

\section{Statistical Analysis}

Multivariate logistic regression models were constructed to identify variables that were independently associated with vaccine uptake among high risk individuals. To build a logistic regression model, a set of demographic and medical predictors was initially used with enter selection, adjusted for age group and gender, due to their theoretical significance and afterwards a backward elimination process to define a significant variables model. Odds ratios (ORs) are reported with their respective 95\% confidence intervals $(95 \% \mathrm{CI})$. Variables included in the multivariate analysis were those that can have effect on vaccination uptake, as reported in the bibliography. Multiple logistic regression models were constructed to identify independent factors associated with vaccination status. Variables that were significant in the bivariate analyses were included in the logistic regression model.

All statistical analyses were performed using the complex samples module of SPSS 21 (IBM, SPSS Statistics). Results were considered statistically significant when the p-value was less than 0.05 .

\section{RESULTS}

A total of 1,168 patients were recruited during the study period, with 828 cases considered as valid, giving a response rate of $70.9 \%$. The age range of participants was from 17 to 102 years (mean 69.08 years, SD 12.397). More than one third of patients $(\mathrm{n}=288,34.8 \%)$ were vaccinated against seasonal influenza, while $540(65.2 \%)$ of them were not.

The majority of the participants $(81.4 \%)$ were over 60 years old. Among the participants 555 (47.5\%) were male, with the number of enrolled men estimated significantly different from the number of enrolled women $(p<0.05)$. Cardiovascular disease and diabetes mellitus were identified in higher proportion within the patients presented to the health centre. Most of the participants were suffering from 1 to 3 diseases and visited the centre 1 or 2 times per year (Table1).

Table 1. Characteristics of the study sample

\begin{tabular}{|l|c|c|c|}
\hline & $\begin{array}{c}\text { Total } \\
\text { N }\end{array}$ & $\mathbf{n}$ & $\%$ \\
\hline Older than 60 years old & 1,115 & 908 & 81.4 \\
\hline Gender & 1,168 & \multicolumn{2}{|c|}{} \\
\hline Male & & 555 & 47.6 \\
\hline Female & & 613 & 52.4 \\
\hline Chronic respiratory disease & 1,149 & 99 & 8.6 \\
\hline Chronic obstructive pulmonary disorder & & 73 & 6.4 \\
\hline Asthma & & 17 & 1.5 \\
\hline Other respiratory condition & & 9 & 0.8 \\
\hline Diabetes mellitus & 1,149 & 271 & 23.6 \\
\hline Cardiovascular disease & 1,149 & 511 & 44.5 \\
\hline Chronic neurological disease & 1,149 & 75 & 6.5 \\
\hline Chronic kidney disease & 1,148 & 21 & 1.8 \\
\hline Comorbidity & 1,117 & \multicolumn{2}{|l}{} \\
\hline 1 to 3 diseases & & 786 & 68.3 \\
\hline More than 4 diseases & & 331 & 28.9 \\
\hline No. of visits per year & 1,168 & \multicolumn{3}{|l|}{} \\
\hline 1 to 2 times & & 923 & 79.0 \\
\hline 3 to 5 times & & 245 & 21.0 \\
\hline Smokers & 151 & 18.1 \\
\hline
\end{tabular}


Table 2. Univariate analysis of determinants of influenza vaccination

\begin{tabular}{|c|c|c|c|c|}
\hline & \multirow{2}{*}{$\begin{array}{c}\text { Total } \\
\mathrm{N}\end{array}$} & \multicolumn{2}{|c|}{ Vaccinated } & \multirow{2}{*}{$\mathrm{p}$-value } \\
\hline & & $\mathrm{n}$ & $\%$ & \\
\hline \multicolumn{5}{|l|}{ Gender } \\
\hline Male & 397 & 156 & 39.3 & \multirow{2}{*}{0.005} \\
\hline Female & 431 & 132 & 30.6 & \\
\hline \multicolumn{5}{|l|}{ Age categories } \\
\hline Younger than 60 years & 139 & 19 & 13.7 & \multirow{3}{*}{$<0.001$} \\
\hline 60 to 79 years & 514 & 201 & 39.1 & \\
\hline Older than 80 years & 164 & 64 & 39.0 & \\
\hline \multicolumn{5}{|l|}{ Comorbidity } \\
\hline 1 to 3 diseases & 527 & 137 & 26.0 & \multirow{3}{*}{$<0.001$} \\
\hline 4 to 6 diseases & 257 & 130 & 50.6 & \\
\hline More than 6 diseases & 34 & 15 & 44.1 & \\
\hline \multicolumn{5}{|l|}{ No. of visits per year } \\
\hline 1 to 2 times & 602 & 184 & 30.6 & \multirow{2}{*}{$<0.001$} \\
\hline 3 to 5 times & 220 & 102 & 46.4 & \\
\hline Non-smokers & 673 & 244 & 36.3 & \multirow{2}{*}{0.041} \\
\hline Smokers & 148 & 42 & 28.4 & \\
\hline
\end{tabular}

A statistically significant difference was found between vaccinated and unvaccinated patients when gender factor was checked $(p=0.005)$. An analysis of the data revealed that younger patients ( $\leq 80$ years) were vaccinated as often as those older than 80 years $(\mathrm{p}<0.005)$, while patients with coexistence of 4-6 diseases were vaccinated at a $50.6 \%$ rate $(\mathrm{p}<0.001)$. As seen in Table 2 , almost one third ( $n=42,28.4 \%)$ of smokers have received the influenza vaccine.

There were significant differences in influenza vaccination rate among the study's medical condition subgroups. Among the predisposing diseases, the highest immunization rate was observed in those suffering from chronic kidney disease (55.6\%), although this was not statistically significant $(p=0.790)$. Other non-significant factors in the bivariate model were patients with malignancies and neurological diseases. Considering enabling factors, significant predictors were all the examined factors, i.e. gender, age, number of visits annually, comorbidity, and smoking. Significant healthneed factors in the bivariate model for increased vaccination rate uptake were the presence of a cardiovascular disease (CVD) diagnosis, respiratory diseases and diabetes mellitus (Table 3).

When we adjusted the multivariate model for age and gender among the predisposing factors, an age between 60-79 years was the strongest predictor for receiving vaccine $(\mathrm{OR}=2.95,95 \% \mathrm{CI}$ : $1.71-5.10, \mathrm{p}<0.005)$, compared to younger ages, as well as $3-5$ visits to the unit per year $(\mathrm{OR}=1.6,95 \% \mathrm{CI}: 1.13-2.26, \mathrm{p}=0.008)$, compared with fewer or more visits; male gender also increased the likelihood of vaccination $(\mathrm{OR}=1.38,95 \% \mathrm{CI}$ : $1.02-1.86$, $\mathrm{p}=0.036)$, but suffering less than 4 diseases reduced the likelihood of vaccination $(\mathrm{OR}=0.16,95 \% \mathrm{CI}$ : $0.03-0.79, \mathrm{p}=0.025)$, compared with those that were not referred any disease. Current smoking status also reduced the vaccination uptake $(\mathrm{OR}=0.8$, $95 \%$ CI: $0.52-1.24)$, but this finding was not statistically significant $(\mathrm{p}=0.330)$.
Table 3. Univariate analysis for vaccination in high risk medical condition patients

\begin{tabular}{|l|c|c|c|c|}
\hline \multirow{2}{*}{} & Total & \multicolumn{2}{|c|}{ Vaccinated } & \multirow{2}{*}{ p-value } \\
\cline { 2 - 4 } & $\mathbf{N}$ & $\mathbf{n}$ & $\%$ & \\
\hline Chronic respiratory disease & 76 & 41 & 53.9 & \multirow{2}{*}{$<0.001$} \\
\hline COPD & 57 & 36 & 63.2 & \\
\hline Asthma & 12 & 4 & 33.3 & \\
\hline Diabetes & 224 & 91 & 40.6 & 0.033 \\
\hline Cardiovascular disease & 403 & 176 & 43.7 & $<0.001$ \\
\hline Chronic neurological disease & 60 & 20 & 33.3 & 0.888 \\
\hline Chronic kidney dysfunction & 18 & 10 & 55.6 & 0.790 \\
\hline Malignancy & 32 & 13 & 40.6 & 0.298 \\
\hline
\end{tabular}

COPD - chronic obstructive pulmonary disorder

When we examined the role of medical factors in the same model, it was observed that the presence of conditions such as chronic respiratory disease $(\mathrm{OR}=2.24,95 \% \mathrm{CI}$ : $1.32-3.81$, $\mathrm{p}=0.003)$ and $\operatorname{CVD}(\mathrm{OR}=1.47,95 \% \mathrm{CI}: 1.02-2.12, \mathrm{p}=0.038)$ increased the likelihood of vaccination among individuals. The presence of diabetes, a malignancy, renal and neurological disorders did not seem to contribute significantly in the vaccination uptake $(\mathrm{OR}=1.06,95 \% \mathrm{CI}: 0.74-1.23, \mathrm{p}=0.741 ; \mathrm{OR}=1.15,95 \%$ CI: $0.53-2.5, p=0.732 ; \mathrm{OR}=1.54,95 \% \mathrm{CI}: 0.06-4.22, \mathrm{p}=0.397$; $\mathrm{OR}=0.89,95 \% \mathrm{CI}: 0.47-1.68, \mathrm{p}=0.721$, respectively). A regression model with backward elimination process, confirmed that a significant contribution to the model seems to have fewer predictors (Table 4). The most contributing factor appeared to be an age over 60, with the age group of 60 to 79 as the most likely to be annually vaccinated $(\mathrm{OR}=3.08,95 \% \mathrm{CI}: 1.791-5.291$, $\mathrm{p}<0.005$ ), compared to younger ages. An age over 80 years had a similar effect $(\mathrm{OR}=2.91,95 \% \mathrm{CI}: 1.58-5.37, \mathrm{p}=0.001)$. Other factors were 3 to 5 annual visits $(\mathrm{OR}=1.579,95 \% \mathrm{CI}$ : 1.121-2.224, $\mathrm{p}=0.002$ ), while the coexistence of $1-3$ diseases reduced the likelihood ratio for vaccine uptake $(\mathrm{OR}=0.157$, 95\% CI: $0.031-0.786, p=0.024)$. Medical conditions that seem to affect influenza vaccination were chronic respiratory diseases

Table 4. Odds ratios and $95 \%$ confidence intervals from logistic regression with backward selection of the most contributing factors for influenza vaccination uptake

\begin{tabular}{|l|c|c|c|}
\hline & OR & $95 \%$ Cl & p-value \\
\hline Gender & 1.333 & $0.978-1.816$ & 0.069 \\
\hline Male & 3.078 & $1.791-5.291$ & $<0.001$ \\
\hline Age group & 2.912 & $1.580-5.367$ & 0.001 \\
\hline $60-79$ years & 0.157 & $0.031-0.786$ & 0.024 \\
\hline Older than 80 years & 1.579 & $1.121-2.224$ & 0.009 \\
\hline Comorbidity & 1 to 3 diseases & $1.337-3.795$ & 0.002 \\
\hline No. of visits per year 3 to 5 times & 1.467 & $1.025-2.101$ & 0.036 \\
\hline Chronic respiratory diseases & 2.252 & \\
\hline Cardiovascular diseases & 1.46 &
\end{tabular}


$(\mathrm{OR}=2.25,95 \% \mathrm{CI}: 1.337-3.795, \mathrm{p}=0.002)$ and cardiovascular disease $(\mathrm{OR}=1.46,95 \% \mathrm{CI}: 1.025-2.101, \mathrm{p}=0.036)$.

\section{DISCUSSION}

This study assessed influenza vaccination rate in adulthood at the time of the survey. Data from Sweden show that vaccination rate in general population was $9.3 \%$ for the $2014 / 15$ period, thus $23.2 \%$ of patients with multiple medical conditions were vaccinated, with $18 \%$ vaccination rate for individuals aged 65 and over (13). The uptake in Poland for people over 65 was $14 \%$ in 2007 (14), while in Spain it was 40\% in 2014 (15). In Greece, the available data on vaccination coverage show that $11.4 \%$ of people that suffered from flu-like symptoms were vaccinated with seasonal influenza vaccine in the 2013/14 season (16). In this study, it was found that the rate of influenza vaccination among patients belonging to high risk groups was only $37.9 \%$. This rate is far below the US Healthy People 2020 goal of $90 \%$ vaccination rate for at-risk populations (17) but is higher than shown in other similar studies from Sweden, Poland and Austria $(13,14,18)$, and similar to other Mediterranean countries $(1,15$, 16). However, those rates are still unsatisfactory and below the recommended guidelines of both the WHO and the Council of the EU recommendations. On the other hand, reported percentage in the US is much higher (19).

Our study demonstrated that male individuals seemed to be vaccinated at a higher rate compared to females, which is in stark contrast with previous studies $(14,20)$. No differences in vaccination rates were observed in patients between 60 to 79 years of age compared to those over 80 years. However, this finding cannot be readily compared to other studies, since the majority of them examined individuals over 65 years old $(3,9,10,21)$. Patients visiting the PHC four to six times per year for health consultation appeared to be the most vaccinated, surpassing even patients who reported more than six visits. This can be attributed to the fact that the patient group of less frequent visits eventually demonstrates a greater consistency with their medical appointments. This can be explained by the fact that it is quite common in Greece to visit a family doctor every three months in order to obtain prescribed medication leading to a better compliance of patients with their therapy, certainly higher than in the patients who attend PHC more frequently. Additionally, other authors suggested that vaccinated adults are more likely to have a primary care provider than unvaccinated adults (22), whereas health services utilization seemed to have had no effect on vaccination uptake (21). A striking outcome was that co-existence of up to 3 diseases was found to be a significant predictor of avoiding influenza vaccination.

Other medical conditions that were referred might not place individuals in a high risk group for influenza vaccination conditions, such as hypertension or high blood cholesterol level with no other comorbidities, thyreopathy, eye disorders, prostate enlargement, etc. This can be explained by the fact that these patients are presented only to a single healthcare provider, or to specialists that are not familiar with the annual vaccination schedule.

Data showed that less than one third of smokers were vaccinated, which is important since smokers represent a high risk group for serious flu infection. Smoking was found to be an insignificant predictor for non-vaccination, which firmly confirms the results of previous studies $(15,23,24)$. In this study, current smokers were less likely to be vaccinated for influenza, compared to non-smokers.

Regarding medical conditions that stratify a patient to the high risk group, it appears that patients suffering with chronic obstructive pulmonary disorder (COPD) were vaccinated more frequently, which verified results from other researchers (25). Respiratory distress was found to be the most significant predictor for vaccination in the present study. All patients with other medical conditions were vaccinated at a level below $50 \%$. Another statistically significant factor for vaccination uptake seems to be the presence of cardiovascular disease. The influenza vaccine uptake among cardiovascular patients rose to $43.7 \%$. In comparison, vaccine uptake is $52 \%$ in Spain (15), $50.5 \%$ in the USA for patients with heart conditions, and $66.7 \%$ in Korea $(26,27)$.

Pharmacists have an important role to promote preventative healthcare measures to high risk patients. Influenza vaccination is one of these measures for which pharmacists may play a crucial role as they can identify high risk patients and educate them about the importance of the influenza vaccination. Unfortunately, there were no data on vaccination covered by pharmacists, as this action is nowhere reported or registered.

Our data demonstrate that there is an important disparity of immunization coverage, and the ultimate target of $95 \%$ population coverage is yet to be achieved. Reported factors of non-vaccination in high risk groups were the perception that the vaccine might not be safe or effective, relatively mild nature of the flu, self-belief that an individual does not belong to a high risk group, as well as a general distrust in vaccination, a dread of toxicity from some vaccine components or excipients, and an overall fright of adverse reactions $(28,29)$. A possible explanation for the poor vaccination rates could be the lack of national strategies in promoting vaccination and the lack of compensation for healthcare practitioners to administer the vaccine.

Important strengths of this study were the very high participation rate of patients, as well as the fact that from the primary health care view in Greece, this survey is among the first to evaluate the influenza vaccination rate among patients belonging to high risk groups.

On the other hand, among limitations of our study was the fact that we could not establish causality between the different factors and influenza vaccination due to the cross-sectional nature of the study. In addition, the results may certainly be subject to a recall bias by respondents. Vaccination status was only assessed by self-reporting and not by validation through a medical record. However, previous studies have shown that there is a high level of agreement between self-reports and medical records on influenza vaccination, minimizing the effect of this limitation (30).

\section{CONCLUSION}

Data from this study showed low levels of influenza vaccination among individuals with chronic diseases presented to PHC in Greece. Age groups among 60-79 years are more likely to be vaccinated than younger or older individuals. Also there was no difference between vaccination preference among people with different sex. Despite the repeated recommendations of health professionals for vaccination coverage of patients with chronic 
diseases, there is relatively small number of people vaccinated. Patients in high risk groups need to be informed appropriately and more extensively. Primary health care providers should implement new and more effective strategies to educate vulnerable groups to reduce the incidence of influenza and its complications. Generally, new and more effective strategies will have to be implemented in the $\mathrm{PMC}$, requiring better programming.

\section{Acknowledgements}

The authors gratefully thanks Prof. Frank Sofras, MD, for providing language help and proof-reading of the article.

\section{Conflict of Interests}

None declared

\section{REFERENCES}

1. Sessa A, Rossi A, Cricelli I. Adult immunization schedule. The general practitioner's perspective and new tools for better practice. J Prev Med Hyg. 2015;56(1):E9-11.

2. World Health Organization. Influenza seasonal. Fact sheet no. 211 [Internet]. Geneva: WHO; 2003 [cited 2017 Sep 6]. Available from: http:// www.who.int/mediacentre/factsheets/2003/fs211/en/.

3. Nicoll A, Ciancio B, Tsolova S, Blank P, Yilmaz C. The scientific basis for offering seasonal influenza immunisation to risk groups in Europe. Euro Surveill. 2008;13(43):19018. doi: 10.2807/ese.13.43.19018-en.

4. Osterholm MT, Kelley NS, Sommer A, Belongia EA. Efficacy and effectiveness of influenza vaccines: a systematic review and meta-analysis. Lancet Infect Dis. 2012;12(1):36-44.

5. World Health Organization. Seasonal vaccination policies and coverage in the European Region [Internet]. Geneva: WHO; 2014 [cited 2017 Sep 6]. Available from: http://www.euro.who.int/en/health-topics/ communicable-diseases/influenza/vaccination/seasonal-vaccinationpolicies-and-coverage-in-the-european-region.

6. Ohmit SE, Thompson MG, Petrie JG, Thaker SN, Jackson ML, Belongia EA, et al. Influenza vaccine effectiveness in the 2011-2012 season: protection against each circulating virus and the effect of prior vaccination on estimates. Clin Infect Dis. 2014;58(3):319-27.

7. Kissling E, Valenciano M, Buchholz U, Larrauri A, Cohen JM, Nunes B, et al. Influenza vaccine effectiveness estimates in Europe in a season with three influenza type/subtypes circulating: the I-MOVE multicentre casecontrol study, influenza season2012/13. Euro Surveill. 2014;19(6):20701. doi: 10.2807/1560-7917.es2014.19.6.20701

8. Georgakopoulou T, Menegas D, Katsioulis A, Theodoridou M, Kremastinou J, Hadjichristodoulou C. A cross-sectional vaccination coverage study in preschool children attending nurseries-kindergartens: implications on economic crisis effect. Hum Vaccin Immunother. 2017;13(1):190-7.

9. Warner JG, Portlock J, Smith J, Rutter P. Increasing seasonal influenza vaccination uptake using community pharmacies: experience from the Isle of Wight, England. Int J Pharm Pract. 2013;21(6):362-7.

10. Isenor JE, Killen JL, Billard BA, McNeil SA, MacDougall D, Halperin $\mathrm{BA}$, et al. Impact of pharmacists as immunizers on influenza vaccination coverage in the community-setting in Nova Scotia, Canada: 2013-2015. J Pharm Policy Pract. 2016;9:32. doi: 10.1186/s40545-016-0084-4.

11. Arabyat RM, Raisch DW, Bakhireva L. Influenza vaccination for patients with chronic obstructive pulmonary disease: implications for pharmacists. Res Social Adm Pharm. 2018;14(2):162-9.

12. Charan J, Biswas T. How to calculate sample size for different study designs in medical research? Indian J Psychol Med. 2013;35(2):121-6.

13. Leval A, Hergens MP, Persson K, Örtqvist $\AA$. Real-time real-world analysis of seasonal influenza vaccine effectiveness: method develop- ment and assessment of a population-based cohort in Stockholm County, Sweden, seasons 2011/12 to 2014/15. Euro Surveill. 2016;21(43):30381. doi: 10.2807/1560-7917.ES.2016.21.43.30381

14. Nitsch-Osuch A, Wardyn K. Influenza vaccine coverage in age-related risk groups in Poland, 2004-2007. Cent Eur J Public Health. 2009; 17(4):198202.

15. Astray-Mochales J, de Antres AL, Hemandez-Barrera V, RodriguezRieiro C, Garrido PC, Esteban-Vasallo MD, et al. Influenza vaccination coverages among high risk subjects and health care workers in Spain. Results of two consecutive National Health Surveys (2011-2014). Vaccine. 2016;34(41):4898-904.

16. Lytras T, Kossyvakis A, Melidou A, Andreopoulou A, Exindari M, Gioula $\mathrm{G}$, et al. Influenza vaccine effectiveness against laboratory confirmed influenza in Greece during the 2013-2014 season: a test negative study. Vaccine. 2015;33(2):367-73.

17. National Center for Health Statistics. Healthy people 2010: general data issues [Internet]. CDC; 2015 [cited 2017 Sep 6]. Available from: https:// www.cdc.gov/nchs/data/hpdata2010/hp2010_general_data_issues.pdf.

18. Kunze U, Kunze M. The Austrian vaccinationparadox: tick-borne encephalitis vaccination versus influenza vaccination. Cent Eur J Public Health. 2015;23(3):223-6.

19. Norris T, Vahratian A, Cohen RA. Vaccination coverage among adults aged 65 and over: United States, 2015. NCHS Data Brief. 2017;(281):1-8.

20. Marshall E, Salmon D, Bousfiha N, Togola Y, Ouedraogo F, Santantonio $\mathrm{M}$, et al. Vaccination coverage among social and healthcare workers in ten countries of Samu-social international sites. Vaccine. 2017;35(39):5291-6.

21. Fabiani M, Riccardo F, Di Napoli A, Gargiulo L, Declich S, Petrelli A. Differences in influenza vaccination coverage between adult immigrants and Italian citizens at risk for influenza-related complications: a crosssectional Study. PLoS One. 2016;11(11):e0166517. doi: 10.1371/journal. pone. 0166517

22. Sutcliffe K, Kilgore PE, DeHoff K, Evans R, Kaye KS, Malosh RE, et al. Survey of vaccination knowledge and acceptance among adults admitted to an urban emergency department. Vaccine. 2017;35(8):1148-51.

23. Burns VE, Ring C, Carroll D. Factors influencing influenza vaccination uptake in an elderly, community-based sample. Vaccine. 2005;23(27):3604-8

24. Pearson WS, Dube SR, Ford ES, Mokdad AH. Influenza and pneumococcal vaccination rates among smokers: data from the 2006 Behaviora Risk Factor Surveillance System. Prev Med. 2009;48(2):180-3.

25. Nitsch-Osuch A, Gołębiak I, Wyszkowska D, Rosińska R, Kargul L, Szuba B, et al. Influenza vaccination coverage among Polish patients with chronic diseases. Adv Exp Med Biol. 2017;968:19-34.

26. Lu PJ, O'Halloran A, Ding H, Srivastav A, Williams WW. Uptake of influenza vaccination and missed opportunities among adults with highrisk conditions, United States, 2013. Am J Med. 2016;129(6):636.e1-636. e11. doi: 10.1016/j.amjmed.2015.10.031.

27. Yang TU, Song JY, Noh JY, Cheong HJ, Kim WJ. Influenza and pneumococcal vaccine coverage rates among patients admitted to a teaching hospital in South Korea. Infect Chemother. 2015;47(1):41-8.

28. Sypsa V, Livanios T, Psichogiou M, Malliori M, Tsiodras S, Nikolakopoulos I, et al. Public perceptions in relation to intention to receive pandemic influenza vaccination in a random population sample: evidence from a cross-sectional telephone survey. Euro Surveill. 2009;14(49):19437. doi: 10.2807/ese.14.49.19437-en.

29. Dáňová J, Šálek J, Kocourková A, Čelko AM. Factors associated with parental refusal of routine vaccinationin the Czech Republic. Cent Eur J Public Health. 2015;23(4):321-3

30. Mangtani P, Shah A, Roberts J. Validation of influenza and pneumococcal vaccine status in adults based on self-report. Epidemiol Infect. 2007;135(1):139-43. 